\title{
Guest editorial: special issue on diagnosis, opacity and supervisory control of discrete event systems
}

\author{
Christos G. Cassandras ${ }^{1} \cdot$ Alessandro Giua $^{2}$
}

Published online: 26 April 2018

C 2018 Springer Science+Business Media, LLC, part of Springer Nature

It is an established tradition of the Journal of Discrete Event Dynamic Systems (J-DEDS) to publish every 2 years a special issue devoted to advances in the area of Discrete Event Systems (DES). For this issue, which was announced in 2016, we selected two broad topics that are central within our research community, namely performance analysis and optimization and diagnosis, opacity and supervisory control. New original contributions in these two areas have recently appeared and it was our goal to take stock of the state of the art and explore new research directions that may be fruitfully pursued over the next several years. After consultation with colleagues following the last International Workshop on DES (WODES 2016, May 2016 in Xi'an, China) we invited seventeen groups of authors to submit papers for these two special issues. These papers were reviewed according to the normal review process of JDEDS and thirteen of them were finally accepted.

The first of the two special issues, entitled "Performance Analysis and Optimization of Discrete Event Systems," contained six papers and appeared in March 2018 (Vol. 28, No. 1).

Here we are introducing the second issue, entitled "Diagnosis, Opacity and Supervisory Control of Discrete Event Systems," which contains seven papers. A brief description of each paper follows.

Tong, Li, Seatzu and Giua in "Current-state opacity enforcement in discrete event systems under incomparable observations" present an approach to design a supervisor for enforcing current-state opacity on finite automata, assuming the intruder is not aware of the supervisor. They consider a general case in which the intruder and the supervisor may observe different and unrelated sets of events.

Fabre, Hélouët, Lefaucheux and Marchand in "Diagnosability of repairable faults" address issues of diagnosis and diagnosability for finite automa with non-persistent faults, i.e., faults that can appear and be repaired. They prove that in this setting checking for diagnosability is PSPACE-complete and show that faults can be reliably counted provided the system is diagnosable for faults and for repairs.

Alessandro Giua giua@diee.unica.it

1 Boston University, Boston, MA 02215, USA

2 University of Cagliari, Cagliari, CA, Italy 
Nunes, Moreira, Alves, Carvalho and Basilio in "Codiagnosability of networked discrete event systems subject to communication delays and intermittent loss of observation" consider diagnosis and diagnosability for finite automa in a decentralized setting where communications occurring in the network connecting local diagnosers and sensors may be affected by delays or intermittent loss of observation. They also present necessary and sufficient conditions for a language to be network codiagnosable and an algorithm to verify this property.

Ammour, Leclercq, Sanlaville and Lefebvre in "Faults prognosis using partially observed stochastic Petri-nets: an incremental approach" deal with the problem of fault prognosis in stochastic discrete event systems represented by partially observed stochastic Petri nets. They propose an incremental procedure that, based on a timed measurement trajectory, computes the probability that a fault will occur in a future time interval.

Cai, Zhang and Wonham in "Characterizations and effective computation of supremal relatively observable sublanguages" present a new characterization of relative observability, based on which an operator on languages is proposed whose largest fixpoint is the supremal relatively observable sublanguage. Moreover, for the purpose of control, they also propose a second operator that in the regular case computes the supremal relatively observable and controllable sublanguage.

Keroglou and Hadjicostis in "Probabilistic system opacity in discrete event systems" introduce an original notion of opacity considering a setting where a user selects a specific hidden Markov model out of a given set but would like to "hide" the chosen system from eavesdroppers. They also present a necessary and sufficient condition to guarantee that the intruder cannot achieve a pre-assigned level of certainty about which model has been selected.

Finally, Takai and Kumar in "Implementation of inference-based diagnosis: computing delay bound and ambiguity levels" consider a decentralized setting in which each diagnoser uses not only its own knowledge of the system behavior, but also its inference about the possible knowledge of the system behavior of other diagnosers to arrive at its own local decision. This scheme requires knowing the bounds for the delay between a fault occurrence and it diagnosis and, among other results, the paper presents on approach for the offline computation of these bounds.

As a conclusion, we would like to thank all the authors for their valuable contributions to the two special issues, the associate editors of J-DEDS who managed the reviewing process and the reviewers who made many helpful suggestions to improve the papers.

Guest Editors

Christos G. Cassandras and Alessandro Giua 